NASA Technical Memorandum 103167

AIAA-90-2417

\title{
A Modeling Technique for STOVL Ejector and Volume Dynamics
}

C.K. Drummond and W.S. Barankiewicz

Lewis Research Center

Cleveland, Ohio

(NASA-TM-103167) A MODELING TECHNIOUE FOR.

$N 90-2 ? 550$

STUYL EJECTOK ANO VOLUME DYNAMICS (NASA)

$13 \mathrm{P}$

CSCL PIE

\section{Unclas}

Prepared for the

26th Joint Propulsion Conference

cosponsored by the AIAA, SAE, ASME, and ASEE

Orlando, Florida, July 16-18, 1990 


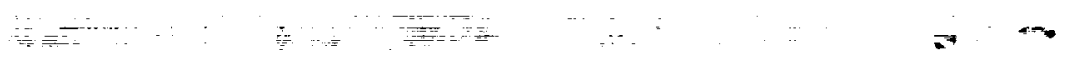




\title{
A MODELING TECHNIQUE FOR STOVL EJECTOR AND VOLUME DYNAMICS
}

\author{
C. K. Drummond ${ }^{*}$ and W. S. Barankiewicz ${ }^{* *}$ \\ National Aeronautics and Space Administration \\ Lewis Research Center \\ Cleveland, Ohio 44135
}

\section{Abstract}

New models for thrust augmenting ejector performance prediction and feeder duct dynamic analysis are presented and applied to a proposed STOVL aircraft configuration. Central to the analysis is the nontraditional treatment of the time-dependent volume integrals in the otherwise conventional control-volume approach. In the case of the thrust augmenting ejector, the analysis required a new relationship for transfer of kinetic energy from the primary flow to the secondary flow. Extraction of the required empirical corrections from current steady-state experimental data is discussed; a possible approach for modelling insight through CFD is presented.

\section{Nomenclature}

A cross-sectional area

$b \quad$ jet half-width

$B \quad$ channel half-width

C) empirical coefficient

d effective nozzle diameter

h enthalpy

$K F \quad$ kinetic energy

$m$ mass flowrate

$m_{c e} \quad$ control volume mass

i. momentum flux

$M_{c \nu} \quad$ control volume momentum

$M_{1 P} \quad$ Mach number of primary nozzle flow

$P \quad$ pressure

$t \quad$ time

v. velocity

I. volume

IV unit depth of control volume

$x \quad$ transverse coordinate

$z \quad$ streamwise coordinate

$p$ density

中 self-similar profile function

$\sigma \quad$ turbulent flow constant

z dimensionless transverse coordinate subscripts

$c v$ control volume

e entrained stream

$m$ primary stream centerline

$1 \mathrm{~s}$ secondary steam at station 1

$1 p \quad$ primary stream at station 1

\footnotetext{
${ }^{*}$ Aerospace Engineer, Member AIAA

${ }^{* *}$ Aerospace Engineer
}

Copyright (C) 1990 by the American Institute of Aeronautics and Astronautics, Inc. No copyright is asserted in the

United States under Title 17, U.S. Code. The U.S. Government has a royalty-free license to exercise all rights under the copyright claimed herein for Governmental purposes.

All other rights are reserved by the copyright owner.

\section{Background}

Thrust augmenting ejectors are candidate componenets for powered lift propulsion subsystems of Short Take-Off Vertical Landing (STOVL) aircraft. Figure 1 illust rates that a typical cjector is a mechanically simple device in which a convergent nozzle is placed within a shroud; in the case of STOVL aircraft, the wing and fuselage act as part of the shroud. Except for a valve to control flow through the nozzle feeder duct, no other parts of this system are required to move during ejector operation (portions of the ejector can, however, fold away for forward flight).

Interest in the dynamic response of an ejector system extends from an effort to develop a complete propulsion system simulation for ASTOVL aircraft (Mihaloew and Drummond, 1989; Akhter el. al., 1989). Propulsion system simulations that run in real-time are an important part of research on design methodologies for Integrated Flight and Propulsion Control (IFPC) systems. In this context a useful ejector characteristic to know is the frequency response of ejector thrust output (due to a change in the primary noz:sle flow condition). If the ejector response is outside the bandwidth of the flight control system (see Figure 2) then a simple quasi-steady ejector model is all that is needed for the propulsion system simulation. In such a case the ejector model could be, for instance, a simple table look-up; this approach readily meets real-time simulation constraints (on the AD100 real-time simulation computer, a table look-up consumes about 2 microseconds).

On the other hand, if the ejector response falls within the range of the flight control (or if a new ejector system has an unknown response) a high-fidelity ejector model must be constructed. Since accuracy is typically synonymous with a significant amount of model detail, constructing a transient ejector model to meet the real-time simulation requirement is a fairly difficult task. Also, the need to predict transient ejector performance aggravates a more fundamental modelling problem exlending from an incomplete picture of steady-state cjector phenomena.

For simplicity in discussion and analysis, an ejector system is usually subdivided along functional lines into an inlet, mixing region, and diffuser. Porter and Squires (1981) present a perspective whereby an ejector gas flow undergoes changes analogous to those of a gas turbine: both systems have an inlet and nozzle (diffuser), but in an cjector the compression, combustion (energy addition), and turbine processes are contained within the mixing region. Compression of and energy addition to the secondary flow results from interaction with the primary flow; the energy 
obtained by expansion of the primary nozzle flow is analogous to the energy input a turbine provides. It is interesting that the potential for augmentation of the primary ejector thrust is understood clearly to be a function of the reduced "ambient" pressure the primary nozzle discharge sees; what is less understood is the detailed mechanism of the primary-secondary entrainment phenomenon responsible for that environment. Central to improving our understanding of the interaction between the primary and secondary flow is a better understanding of the physics behind turbulent, compressible flow shear layers (Goebel and Dutton, 1990, provide a recent look at this problem).

\section{CED Approach}

One approach to the ejector analysis is based on first principles and typically blends the mean value equations for momentum, energy and heat with phenomenologial models of turbulence for closure (Anderson et. al., 1984; Townsend, 1980). Finite-difference, finite-element, or spectral methods of analysis are then employed for the discretization and solution of the final system of equations (Peyret and Taylor, 1983). These solution methods are representative of modern Computational Fluid Dynamics (CFD). Although CFD provides a rigorous approach to analysis of the flowfield, it is not expected that real-time solutions are achievable in the forseeable future.

Another problem concerns identification of the appropriate boundary conditions and turbulence model to use for the description of the mixing region. It appears the state-of-the-art provides steady-flow predictions more accurate in an integral sense (pressure, thrust) than pointwise (velocity). For instance, Deese and Agarwal (1988) investigated several CFD analyses (including their own) and remark on the general failure to produce "acceptable" velocity profiles. The capability to predicit thrust is encouraging, but wall shear stress estimates will not be more accurate than the velocities computed. Modern CFD analyses designed to attack these problems is an active area of research (see, for instance, Lowrie, 1990; Choi and Soh, 1990).

\section{Control-Volume Approach}

An alternative to CFD is the control-volume approach. In this case the governing equations express "bulk" conservation of mass, momentum, and energy. Although the control-volume method greatly simplifies the mathematical analysis, the opportunity to introduce a detailed mathematical description of any transport mechanisms is often lost. Real-flow effects are generally introduced through loss factors (nozzle coefficient; wall friction factor) or specified skewness conditions (non-uniform secondary or mixed flow profiles). By default, such corrections are accurate for the model tested, but tend to be configuration dependent and have unknown scale effects. Despite criticizm that control-volume predictions devoid of empirical correction factors do not rigourously account for mixing, entrainment, and boundary-layer effects, this approach has been the foundation for many steady-flow ejector performance predictions exhibiting rapid execution speed of execution and acceptable engineering accuracy. We find this to be an attractive foundation for the exploration of a transient flow simulation.

Here, a transient flow is a 'temporary' unsteady flow, associated with, for example, a change in ejector operation from one steady-state condition to another. Contrast this with oscillatory flows in which a periodic time-asymptotic flow character is exhibited. Ejectors utilizing pulsed primary nozzle flows are of the latter type. In the present simulation the focus on transient, not oscillatory, ejector phenomena descends from flight-critical aircraft flight control scenarios; an example would be transition to forward flight from vertical take-off.

A basic difficulty in application of the control-volume method to the study of transient flows is in the treatment of the time-dependent volume integrals -- unless you consider the flow quasi-steady, it is essential these terms be included in the problem formulation. One solution to this modelling problem to draw in as many assumed features of the flowfield as possible.

\section{Scope of Work}

An empirically based model for the jet mixing turbulent interaction region is explored within the framework of a control volume analysis. Three fundamental assumptions are discussed in the present work that reduce the transient ejector analysis problem to an attractively simple system of equations. We also remark on a control-volume approach to simulation of duct dynamics. In comparison with the ejector analysis, the mathematical treatment is considerably simplified by the absence of a secondary flow. Generally the focus of discussion is on the transient features of the analysis since the treatment of steady-state flows is given in, for instance, Addy and Dutton (1974).

Estimation of the empirical coefficients required in the control-volume method of analysis is discussed. Typical results from the ejector and duct analysis for a proposed STOVL aircraft are presented. Preliminary finite-element CFD results for the ejector mixing region are also presented; CFD is being pursued as one means to augment the ejector modeling process.

\section{Ejector Analysis}

In an unsteady flow problem, considerable mathematical convenience follows when a quasi-steady flowfield response can be assumed -- the time-derivative terms in the governing differential equations can be dropped. At each instant in time the flow is assumed to respond instantly to boundary condition changes. The validity of such an assumption, however, requires the characteristic time of the forcing function to be the same as (or greater than) the relaxation time of the flow. For a given flowfield disturbance, pressure forces respond more quickly than viscous forces, so the former are therefore more appropriately treated as quasi-steady. 
Note that the mixing region shear layer physics are the cause of a situation that yields pressure gradient effects in the inlet and diffuser. It is therefore reasonable to assume the inlet and diffuser behave in a quasi-steady fashion. In the present work the analysis focuses on describing the mixing region characteristics shown in Figure 3.

The development begins with some comments on the mass, momentum, and energy balances for a mixing region control volume. Then, the application of self-similar velocity profiles to the governing equations is presented. In the last section we discuss division of the energy balance into the (usual) thermal energy balance and a new function for mechanical energy.

\section{Mixing Region Control-Volume Equations}

Several assumptions simplify the mixing region mathematical representation without unduly compromising the physics of interest. A one-dimensional flow assumption limits field variable gradients to occur along (but not across) the streamwise axis. Viscous, blockage, and Reynolds number effects are neglected. Gases are assumed thermodynamically perfect (constant specific heat) and described by the equation of state. The primary and secondary flows can be of different total pressure and temperature, but equality of static pressure at the point of confluence of the two jets is enforced. These assumptions are applied to the following integral form of the conservation equations (see Thompson, 1972):

$$
\begin{gathered}
\frac{d m_{c v}}{d t}=-\oint_{A} \rho \mathbf{v} \cdot \mathbf{n} d A \\
\frac{d}{d t} \int_{V} \rho \mathbf{v} d V=-\oint_{A} \rho \mathbf{v}(\mathbf{v} \cdot \mathbf{n}) d A-\oint_{A} \mathbf{n} \cdot(p \mathbf{I}) d A \\
\frac{d}{d t} \int_{V} \rho\left(h+\frac{u^{2}}{2}-\frac{p}{\rho}\right) d V=-\oint_{A} \rho\left(h+\frac{u^{2}}{2}\right) \mathbf{v} \cdot \mathbf{n} d A
\end{gathered}
$$

Equation (3) represents the energy equation derived from the first law of thermodynamics -- this form of the energy balance is known as the general energy equation. Since discontinuities in, for instance, entropy, are not included in this formulation, the present formulation will not effectively describe shock flows. The general energy equation is a balance of energy due to heat and work. The product of the local fluid velocity and the momentum equation (Eqn.2) yields the mechanical energy equation. Subtracting the mechanical energy equation from the general energy equation produces the heat equation, the latter of which is a simple statement of conservation of heat content in the ejector mixing region,

$$
\frac{d}{d t} \int_{V} \rho\left(h-\frac{p}{\rho}\right) d V=-\oint_{A} \rho h \mathbf{v} \cdot \mathbf{n} d A
$$

In the present work, Eqn.(4) is retained to represent the heat balance, and (as discussed later) a semi-empirical kinetic energy exchange model is substituted for the mechanical energy counterpart.
Some important features of the mixing region flowfield are shown in Figure 3. Primary and secondary potentialcore regions are characterized by a uniform axial velocity (and no transverse component). The inside and outside bounds of the jet shear layer are defined by conditions of, respectively, the primary and secondary potential flows. Momentum transfer from the primary jet results in the eventual consumption of the primary core. Generally the flowfield is considered in a "developing" state prior to the point at which the wall boundary layer meets the outer jet boundary.

\section{Turbulent Jet Approximation}

Extensive experimental data supports the Abramovich(1963) non-dimensional velocity representations of 2-D planar co-flowing free jets:

$$
v=v_{e}(1-\Phi)+v_{m} \Phi=f(\Phi)
$$

where

$$
\Phi(\xi)=\left\{\begin{array}{cc}
\left(1-\xi^{1.5}\right)^{2}, & 0 \leq \xi \leq 1 \\
0, & 1 \leq \xi \leq \xi
\end{array}\right\}
$$

and

$$
\xi=x / b, \quad \xi=B / b
$$

Inclusion of the core region requires a slight modification of these profiles; details are given in Abramovich(1963).

In comparison with the Abramovich free jet approximation, ejectors involve a confined jet in which a streamwise pressure gradient exists. An issue to therefore be resolved is the accuracy to which the Abramovich profiles represent the ejector flow. As a practical matter these self-similar profiles have been used extensively with acceptable accuracy in fluidized bed gas cleaning applications (Donsi et. al., 1980; Tan, 1982). Although the pressure gradient is in the opposite direction (than that of the ejector), the gradients are significant (up to 100\%); this situation encourages the application of Abramovich's profiles outside of his original set of assumptions. A more thorough examination of this approximation appears to be possible if, for instance, raw data from Storms(1989) LDV work or McLachlan et. al. (1984) were curve-fit accordingly.

Since there is a static pressure matching condition at the jet boundary, a uniform transverse pressure distribution is assumed; in the longitudinal (axial) direction a finite pressure gradient exists (Again, this feature partially distinguishes free-jet and confined-jet analyses). This reduces the general pressure profile to the form

$$
\frac{p-p_{e}}{p_{m}-p_{e}}=\Lambda(\xi)=1
$$

To avoid complexity in the use of the ideal gas law, density also has a uniform profile. Since this implies a uniform temperature profile, a compromise has been introduced in the mixing region representation for temperature (Abramovitch suggests the non-dimensional temperature profile be approximated by the square root of the velocity profile). 


\section{Application to the Conservation Equations}

Figure 4 illustrates the finite volume descretization employed in the present work. Since the implicit assumption is that the self-similar profiles are the link between the flow streams in the transverse direction, descretization only occurs in the streamwise direction. To capture a representative variation in flowfield characteristics (Figure 3) it is necessary to divide the mixing region into at least 3 elements. We generalize the analysis by defining a generic element, $k$, bounded by surfaces at $i$ and $j$.

Consider the application of the self-similar profiles to the mass conservation equation. Based on our previous flow assumptions, Equation (1) simplifies to the form

$$
\left(\frac{d m}{d t}\right)_{k}=\dot{m}_{t}-\dot{m}_{i}
$$

where the mass flux is now written

$$
\dot{m}_{t}=\int_{A} \rho_{1} v_{1} d A=2 W \int_{0}^{\xi} \rho_{1} v_{1} d \xi
$$

From which integration over the self-similar profiles yields

$$
\begin{aligned}
\dot{m}_{l} & =2 W b_{1} \rho_{1}\left(0.45 v_{m}+0.55 v_{\mathrm{e}}+(\xi-1) v_{\mathrm{e}}\right)_{1} \\
& =2 W b_{1} Z_{1}
\end{aligned}
$$

The time-derivative term in the mass conservation equation is given by

$$
\left(\frac{d m}{d t}\right)_{k}=2 W B \Delta z\left(\frac{d \rho}{d l}\right)_{1}
$$

where the characteristic density for the finite volume is now approximated by the value of the density at station $j$ (in practice this is a good assumption as long as field variable gradients are "modest" in size). If the characteristic jet expansion width, $b$, also assumes its value at $j$, then substitution and re-arrangement of the continuity equation yields

$$
\left(\frac{d \rho}{d t}\right)_{,}=\frac{b_{1} Z_{1,1}-b_{i} Z_{1, j}}{B \Delta z}
$$

Computation of the jet half-width, $b$, derives from the momentum equation for incompressible flow, applied to the first finite volume; a rectilinear jet expansion is assumed therefrom and compressible flow restored for subsequent calculations (In light of the recent work of Goebel and Dutton (1990), this procedure for computing the jet expansion angle may need to be refined).

When the self-similar profiles are substituted into the momentum balance, equation (2), the jet centerline velocity derivative can be shown to be

$$
\left(\frac{d v_{m}}{d t}\right)_{i}=\frac{\dot{M}_{k}}{2 W b, \Delta z}-\frac{v_{m}+v_{e} F_{1}}{\rho}\left(\frac{d \rho}{d t}\right)_{,}
$$

where

$$
\begin{aligned}
& F_{1}= \frac{\xi-0.45}{0.45} \\
& \dot{M}_{k}= 2 W b_{i}\left(Z_{2, i}+\xi P_{i}\right) \\
&-2 W b_{i}\left(Z_{2, j}+\xi P_{i}\right) \\
& Z_{2}=\int_{0}^{\xi} \rho_{k} v_{k}^{2} d \xi
\end{aligned}
$$

Note in equation (14) that the centerline velocity derivative is a function of the density derivative, equation (13).

Introduction of an ideal gas assumption simplifies the heat equation to the form

$$
\frac{d}{d t} \int p d V=-\oint_{A} p y \mathbf{v} \cdot \mathbf{n} d A
$$

Non-dimensional velocity and pressure profiles provide the pressure derivative result

$\left(\frac{d P}{d t}\right)_{k}=\gamma \frac{b_{k} Z_{1}+b_{k+1} Z_{1}}{\Delta z B}+(\gamma-1) \frac{b_{k} Z_{0}-b_{k+1} Z_{0}}{\Delta z B}$

where $Z_{l}$ has been derived previously and $Z_{0}$ is given at each station by

$$
Z_{0, i}=\int_{0}^{\xi} p_{1} \wedge d \xi
$$

Equations (13), (14), and (19) are the three differential equations required for computing the time derivatives of gas density, pressure, and jet centerline velocity in the ejector mixing region. Closure for the problem requircs definition of the entrained flow state.

\section{Entrained Kinetic Energy Augmentation}

Analysis of the primary and secondary flow interaction has not, to this point, been completed. By themselves, the self-similar profiles close the loop for steady-state flows, but not transient ones. This section provides an approximation for the turbulent flow kinetic energy exchange mechanism to characterize the influence of primary flow changes on the secondary flow. Knowledge of the total change in secondary flow kinetic energy permits updates to the secondary flow state during integration of the field variable time derivatives.

Computations for a specified steady-state condition show that the change in kinetic energy due to mixing is not the same for the secondary flow as it is for the primary. In fact, the gain in kinetic energy of the secondary flow is entirely due to the mixing process, while the mixing loss of the primary flow is only a fraction of its total loss. In balance, the total change of kinetic energy of the primary flow is greater than that of the secondary flow.

In the works of Chow and Addy(1964) and Korst and Chow(1966) the relationship between the change in entrained flow kinetic energy and the total primary flow kinetic energy for a shear layer are discussed, and the results can be expressed in the functional form:

$$
\Delta K E_{1 S}=F\left\{K E_{1 P}, \frac{\sigma}{z}\right\}
$$


where it was empirically determined that

$$
\sigma=12\left(1+0.23 M_{1 p}\right)
$$

The difficulty with the kinetic energy function as given above is that it represents a quasi-steady constant-pressure flow approximation and therefore cannot be used in its present form for the transient flow analysis. To entertain local transport of energy, consider the change in secondary flow to be a combination of changes in primary and secondary flow kinetic energies due to mixing alone,

$$
\Delta K E_{1 S}=G\left\{\Delta K E_{1 P, m}, \Delta K E_{1 s, m}, \frac{\sigma}{z}\right\}
$$

where the subscript $m$ denotes the change in kinetic energy due exclusively to mixing. Numerical experiments suggest the form

$$
\Delta K E_{1 S}^{i+\Delta t}=\Delta K E_{1 S, m}^{i+\Delta t}+\Delta K E_{1 P, m}^{\prime} C_{1}\left(\frac{\sigma}{z}\right)^{2}
$$

This engineering approximation results in the introduction of an undetermined constant, $C_{1}$. In the present work the value of the constant is determined by matching transient solution asymptotes with steady-state performance data (an example is presented later).

Computation of the gain in secondary flow kinetic energy due to mixing is given by

$$
\Delta K E_{1 s, m}=\int_{\zeta}^{\xi} \rho v\left(\frac{v^{2}}{2}-\frac{v_{e}^{2}}{2}\right) d \xi
$$

where $\zeta$ defines the jet boundary streamline (for which the primary mass flow through station $i$ is equal to primary mass flow through $j$ ). For the present discussion this dividing streamline position is assumed known; Korst and Chow(1966) discuss the typical approach of analysis. Expanding the equation for the change in kinetic energy yields

$$
\Delta K E_{1 S, m}=W b \rho\left\{\int_{\zeta}^{1}\left(v^{3}-v v_{e}^{2}\right) d \xi+\int_{1}^{\xi}\left(v^{3}-v v_{e}^{2}\right) d \xi\right\}
$$

Substitution of the self-similar profiles into this expression and integrating the result provides

$$
\begin{aligned}
\Delta K E_{1 S . m}= & N W b \rho\left\{v_{e}^{3}\left(H_{1}+H_{5}-F_{3}-F_{4}\right)\right. \\
& \left.+v_{m} v_{e}^{2}\left(H_{3}-F_{1}\right)+v_{m}^{2} v_{e} H_{2}+v_{m}^{3} H_{4}\right\}
\end{aligned}
$$

where the detailed expressions for the integrals $H_{i}$ and $F_{i}$ are given by Drummond(1988). It should be noted that in the present work the integrals are independent of time.

Similar to the way in which the change in secondary flow kinetic energy was computed, the energy loss of the primary flow is given by

$$
\Delta K E_{1 P, m}=\int_{0}^{\xi} \rho v\left(\frac{v^{2}}{2}-\frac{v_{m}^{2}}{2}\right) d \xi
$$

where the limits of integration reflect interest in the domain of the primary jet cross-section.

Evaluation of the integral at station $i$ yields the result

$$
\begin{gathered}
\Delta K E_{1 P, m}=W b \rho\left(v_{\mathrm{e}}^{3} H_{1}+v_{\mathrm{e}} v_{m}^{2} H_{2}+v_{m} v_{\mathrm{e}}^{2} H_{3}+v_{m}^{3} H_{4}\right. \\
\left.-v_{m}^{3} F_{1}-v_{\mathrm{e}} v_{m}^{2} F_{3}\right)
\end{gathered}
$$

The expressions for the integrals implied in $F_{i}$ and $H_{i}$ can be obtained from Drummond(1988).

It is recognized that the mixing length theory of Korst has only been verified for steady-state flow and, furthermore, that a constant pressure assumption was made in his analysis. For the transient ejector work, Korst's theory is simply employed as a springboard for a new functional relation between the primary and secondary flows. Although the detailed form of the function has not been validated, one must admit that the basic model provides a rational foundation for energy exchange and does allow flexibility in future modelling efforts.

\section{Summary}

Density, velocity, and temperature are the unknown ejector mixing region field variables. The proposed control volume analysis asks the sub-region be characterized by an 'entrained flow' node and a 'jet centerline' node. The initial value problem links the two nodes through a form of the integral equations where a self-similar profile assumption has been blended in. To proceed forward in time, then, (a) integrate the three jet centerline equations forward one time step, (b) compute the entrained velocity from the kinetic energy exchange approximation, and (c) update the entrained flow density and pressure by assuming equilibrium with the primary flow.

\section{Duct Analysis}

Feeder ducts and tailpipes are 'large-volume' STOVL propulsion system components whose dynamic response (so called, volume dynamics) are of interest when (a) coupling of static sub-system components is necessary, or (b) gas dynamics effects are potentially significant. A model that satisfies both of these situations is desired for the general ejector system simulation objective.

The proposed model follows directly from the governing equations for mass, momentum, and energy given by Eqns.(1)-(3). All of the simplifying assumptions stated for the ejector are also assumed here.

As before, mass conservation takes the form of a density derivative

$$
\left(\frac{d \rho}{d t}\right)_{k}=\frac{\dot{m}_{i}-\dot{m}_{i}}{V_{k}}=\frac{\dot{m}_{i}-\dot{m}_{i}}{\bar{A} \Delta x}
$$

where an overbar indicates a volume-average quantity. 
Flow momentum changes in ducts are generally attributable to variations in cross-sectional area or the friction associated with real flows; the governing differential equation is a vector expression, but under the assumtion the unit normals point outward from each boundary there results the scalar balance

$$
\frac{d}{d t}(\rho \cup V)_{k}=M_{1}-M_{j}+F
$$

where the scalar form of the momentum is

$$
M=v \dot{m}+P A
$$

In this work an idealized expression for the internal resistance of the duct shown is given by

$$
F=\vec{P} d A \cong \frac{1}{2}\left(P_{i}+P_{j}\right)\left(A_{j}-A_{1}\right)
$$

where the absence of frictional effects should be noted. Carrying through the differentiation in time and rearranging results in the following equation

$$
\left(\frac{d v}{d t}\right)_{k}=\frac{1}{\bar{\rho} \bar{A} \Delta x}\left(M_{i}-M_{j}+\hat{F}\right)-\frac{\bar{v} d \rho}{\bar{\rho} d t}
$$

The characteristic velocity $\bar{v}$ is a weighted average of the inlet and exit control volume velocities

$$
\bar{v}=\xi v_{1}+(1-\xi) v_{1}
$$

where $\xi$ is a weighting parameter ranging in value from 0 to 1.

In the duct analysis the general power equation is used since it includes representations of thermal and kinetic energy,

$$
\frac{d}{d t}\left\{\rho\left(h+\frac{v^{2}}{2}-\frac{p}{\rho}\right)\right\}_{k}=\dot{E}_{i}-\dot{E}_{i}
$$

where

$$
\dot{E}=\rho\left(h+\frac{v^{2}}{2}\right) \mathbf{v} \cdot \mathbf{n} A=\dot{m}\left(h+\frac{v^{2}}{2}\right)
$$

The time-dependent term can be rearranged to read

$\frac{d}{d t}\left\{\rho\left(h+\frac{v^{2}}{2}-\frac{p}{\rho}\right)\right\}_{k}=\left\{\frac{1}{\gamma-1} \frac{d p}{d t}+\rho \bar{v} \frac{d v}{d t}+\frac{v^{2}}{2} \frac{d \rho}{d t}\right\}_{k}$

and from this an expression for the pressure derivative obtained

$$
\frac{\mathrm{I}}{\gamma-1}\left(\frac{d \rho}{d t}\right)_{k}=\left\{E_{i}-E_{i}\right\}-\left\{\bar{\rho} \frac{d v}{d t}+\frac{v^{2}}{2} \frac{d \rho}{d t}\right\}_{k}
$$

Equations (30), (33), and (37) are the required differential equations to integrate for the prediction of duct dynamics.

\section{Results}

A step-change forcing function is a simple yet effective transient for examining dynamic system response. In this work a step-change in primary nozzle flowrate is imagined to be a 'worst-case' scenario for STOVL ejector operation and is therefore considered an appropriate test for the simulation. For demonstration purposes a flowrate stepchange from 18.7 to $21.9 \mathrm{lb} / \mathrm{mec}$ is chosen because (a) experimental steady-state data at each of these operating points is available, and (b) the $17 \%$ change in primary flowrate is well beyond a "small"-perturbation examination (this exercises the system non-linearities). The rectangular mixing region of the proposed STOVL ejector is approximately $1 \mathrm{ft}$ wide (at the throat), $9 \mathrm{ft}$ in depth (along the wing chord axis), and $0.9 \mathrm{ft}$ long. A $260^{\circ} \mathrm{F}$ primary flow is used to entrain air at ambient conditions. A hover condition is assumed (no forward ejector velocity). The feeder duct was assumed to be straight, $15 \mathrm{ft}$ long, and have a constant 1.1 ft diameter.

\section{Ejector Predictions}

With the STOVL mixing region subdivided into five finite-volumes (along the flow axis), the individual control-volume length of $0.18 \mathrm{ft}$ combined with a characteristic mixing region velocity of, say, $500 \mathrm{ft} / \mathrm{s}$ yields a residence time (elapsed time for the primary nozzle flow to reach the diffuser exit plane) for the flow of approximately $0.4 \mathrm{~ms}$. The numerical time step must be less than this value. A estimate of the stability limit value $\Delta t=.12 \mathrm{~ms}$ is given by the Courant approximation $(\Delta t<0.5 \Delta x / u)$. To avoid infringing on this stability limit a computational time step of $0.1 \mathrm{~ms}$ was chosen for the present work. An explicit time stepping scheme is appropriate for this application since the optimal weighting factors for an implicit approach are generally a function of time.

The empirical coefficient $C_{1}$ in the transient analysis is required for calibration of the function for primary-to-secondary kinetic energy exchange, Equation 24. For a step-function change in the primary flowrate, the ejector, initially operating at a steady-state condition, asymptotically approaches a second steady-state. The desired $C_{I}$ value is the one that provides a match between the predicted asymptote and the known thrust at the second state. Thrust predictions are shown in Figure 5; the "best" $C_{1}$ value appears to be between 0.3 and 0.35 .

For a $760 \mathrm{ft} / \mathrm{s}$ initial primary flow velocity, it appears the $1.2 \mathrm{~ms}$ flow residence time is slightly less than half the 3 millisecond interval for the thrust to reach a new maximum. Oscillations in thrust after that point appear to settle in about 5 milliseconds. This profile suggests a second-order frequency response of the ejector. Under a second-order assumption the ejector test case suggests a 0.75 ejector damping ratio and a natural frequency on the order of $300 \mathrm{~Hz}$.

An (initially) unexpected feature of the thrust profile is the dip in thrust immediately following the step-change in primary nozzle efflux. Examination of the field variable profiles reveals this is not a numerical problem, but that the increase in static pressure associated with the instantaneous change in driving flow temporarily reduces the favorable sub-ambient pressure environment (required for a potential in thrust augmentation) at the primary nozzle discharge. After a short period, the shear layer energy transfer 
becomes commensurate with the increase in primary flow energy to overcome this effect, and the response then continues in an intuitively expected manner.

\section{Duct Dynamics}

Transient flow calculations for the ejector feeder duct were based on the flow profile previously described for the ejector primary nozzle. The results shown in Figure 6 indicate the flowrate response time is nearly equal to the flow residence time (no gasdynamic lag). This prediction is intuitively reasonable because of the high flow velocities and relatively small duct volume involved.

\section{Application of CED for Model Refinement}

Two assumptions in the modeling technique that must be explored further are the self-similar profile assumption and the proposed function for the kinetic energy exchange mechanism. One avenue for examining internal flow conditions is to analyse the internal flowfield with CFD. FIDAP, a commercial CFD finite-element analysis code (Reference 11), was chosen for this purpose. Although the code has a time-dependent turbulent flow solution capability, the version of the code employed could not handle compressibility effects. Also, the CPU requirements for unsteady flow solutions limited our investigation to steady flow analysis. These practical restrictions aside, FIDAP was still considered acceptable for obtaining a "snapshot" of the internal ejector flow since a few studies hinted at some success with this approach (Sohn, 1989; Bullock and Haroutunian, 1989).

\section{EIDAP Results}

A simple test case was of interest that would focus on prediction of conditions in the mixing region. For this purpose a simple 2-D turbulent mixing layer configuration was constructed: a primary jet is discharged through a 0.4in high slot into a 4.88 in section (constant area) mixing region. At the time of this writing, converged turbulent flow solutions (using the $k-\epsilon$ turbulence model and upwinding) have only been obtained for a $0.5 \mathrm{ft} / \mathrm{s}$ primary jet velocity. Although this low a velocity limits the direct applicability of the results, some qualitative comments can be made.

The velocity profiles shown in Figure 7a clearly illustrate the potential core region of the primary flow. These transition to a fully developed appearance at the end of the duct (for clarity, only a small region of the Sin by 14 in mixing region is shown). A test of the appropriateness of the Abramovich self-similar profile assumption is shown in Figure $7 \mathrm{~b}$. Turbulent flow profiles at the axial stations $\mathrm{z} / \mathrm{d}=18$ and 30 , and the solution for a laminar flow at $z / d=30$ are plotted against the Abramovich profile. It is interesting to note how closely the laminar flow solution follows the turbulent approximation. For the turbulent flow computation, we observe that the fully-developed profile assumption is clearly more appropriate at $\mathrm{z} / \mathrm{d}=\mathbf{3 0}$ than at 18.
It is evident from these results that it is important to include the potential core in the velocity profile representation. Also, some account of the developing nature of the flow is warranted for $\mathrm{z} / \mathrm{d}<30$.

\section{Summary}

An ejector simulation method that includes ejector transients and with the potential to run real-time has been presented. The finite volume method permits rapid evaluation of the time dependence of field variables in a thrust augmenting ejector mixing region. For complete verification of the proposed modeling technique it is necessary to have available transient ejector performance data to compare with the simulation output. At the present time, however, only steady-state tests have been conducted for the ejector shown in Figure 1; a test plan is in-progress to expand our database to include transient flow experimental data.

Stability problems appear to be indicating a need for improvements in the model for the case of choked primary nozzle flows. Although the present model is valid for compressible flow, entropy changes must be accounted in a more direct way for a proper treatment of shock flow conditions. The self-similar profiles are obviously not appropriate in dealing with such flows.

Concern over the use of a constant value of $C_{1}$ results in its variation with limits on the primary flow. It remains to derive a rational form for a function (instead of a constant) to be used for matching thust at these extremes.

Futher CFD activity is in-progress for computation of higher-speed flows and for a geometry that is more representative of the proposed ejector configuration.

\section{Beferences}

1. Abramovich,G. (1963), The Theory of Turbulent Jets, MIT Press.

2. Addy,A. and Dutton,J. (1974), "Ejector-diffuser theory and experiments," Report No.UILU-ENG-74-4009, University of Illinois at Urbana-Champaign.

3. Akhter, M.M., Vincent, J.H. Berg, D.F., and Bodden, D.S. (1989), "Simulation Development for US/Canada ASTOVL Controls Technology Program," 20th Modeling and Simulation Conference, May 4-5, Pittsburg, Pennsylvania.

4. Anderson, D., Tannehil, J., and Pletcher, R. (1984), Computational Fluid Mechanics and Heat Transfer, New York: McGraw Hill.

5. Bullock, C.E. and Haroutunian, V. (1989), "Analysis of Air Circulation Patterns Within the Sistine Chapel With a Proposed Air Conditioning System," Proceedings of the Third FIDAP Users Conference, September 24-26.

6. Choi, Y. and Soh, S. (1990), "Computational Analysis of the Flowfield of a Two-Dimensional Ejector Nozzle," ALAA-90-1901. 
7. Chow,W.L. and Addy,A.L. (1964), "Interaction between primary and secondary streams of supersonic ejector systems and performance characteristics," ALAA Joumal, V.2, No.4, pp.686-695.

8. Deese, J.E. and Agarwal, R.K. (1988), "A Numerical Study of Viscous Flows in Inlets and Augmentors," ALAA-88-0187.

9. Donsi, G. Massimilla, L. and Colantuoni, L. (1980), "The Dispersion of Axisymmetric Gas Jets in Fluidised Beds," Fluidization, Grace and Matsen (Eds.) Plenum Press: New York.

10. Drummond, C.K. (1988), "A Control-volume method of analysis of unsteady thrust augmenting ejector flows," NASA CR-182203.

11. Fluid Dynamics International (1987), FIDAP, Volumes 1-4, Evanston Illinois.

12. Goebel, S.G. and Dutton, J.C. (1990), "Velocity Measurements of Compressible, Turbulent Mixing Layers," AlAA-90-0709.

13. Korst,H. and Chow,W. (1966), "Non-isoenergetic turbulent jet mixing between two compressible streams at constant pressure," NASA-CR-419.

14. Lowrie, B. (1990), "A Multi-Zone k-E Turbulence Model for Complex Configurations", ALAA-90-2001.
15. McLachlan, B.G., Krothapalli, A. and Nagaraja, K. (1984), "Flow Structure within a Heated Rectangular Jet Ejector," ALAA Paper 84-0571.

16. Mihaloew, J.R. and Drummond, C.K. (1989), "STOVL Aircraft Simulation for Integrated Flight and Propulsion Controls Research," NASA TM-102419.

17. Peyret, R. and Taylor,T. (1982), Computational Methods for Fluid Flow, New York: Springer-Verlag.

18. Porter,J. and Squyers,R. (1981), "A summary/overview of ejector augmentation theory and performance," USAF Technical Report No.R-91100-9CR-47.

19. Sohn, J.L. (1989), "Numerical Analysis of Laminar and Turbulent Incompressible Flows Using the Finite Element 'Fluid Dynamics Analysis Package FIDAP', NASA CR-179390.

20. Storms, B.L. (1989), "An Experimental Study of a Three-Dimensional Thrust Augmenting Ejector Using Laser Doppler Velicometry," NASA CR-177531.

21. Tan, B.K. (1982), "Filtration of Gases in Modile Granular Beds," Ph.D. Dissertation, University of Cambridge (U.K.).

22. Thompson,P. (1972), Compressible Fluid Dynamics, New York: McGraw-Hill.

23. Townsend, A.A. (1980), The Structure of Turbulent Shear Flow, Cambridge University Press.

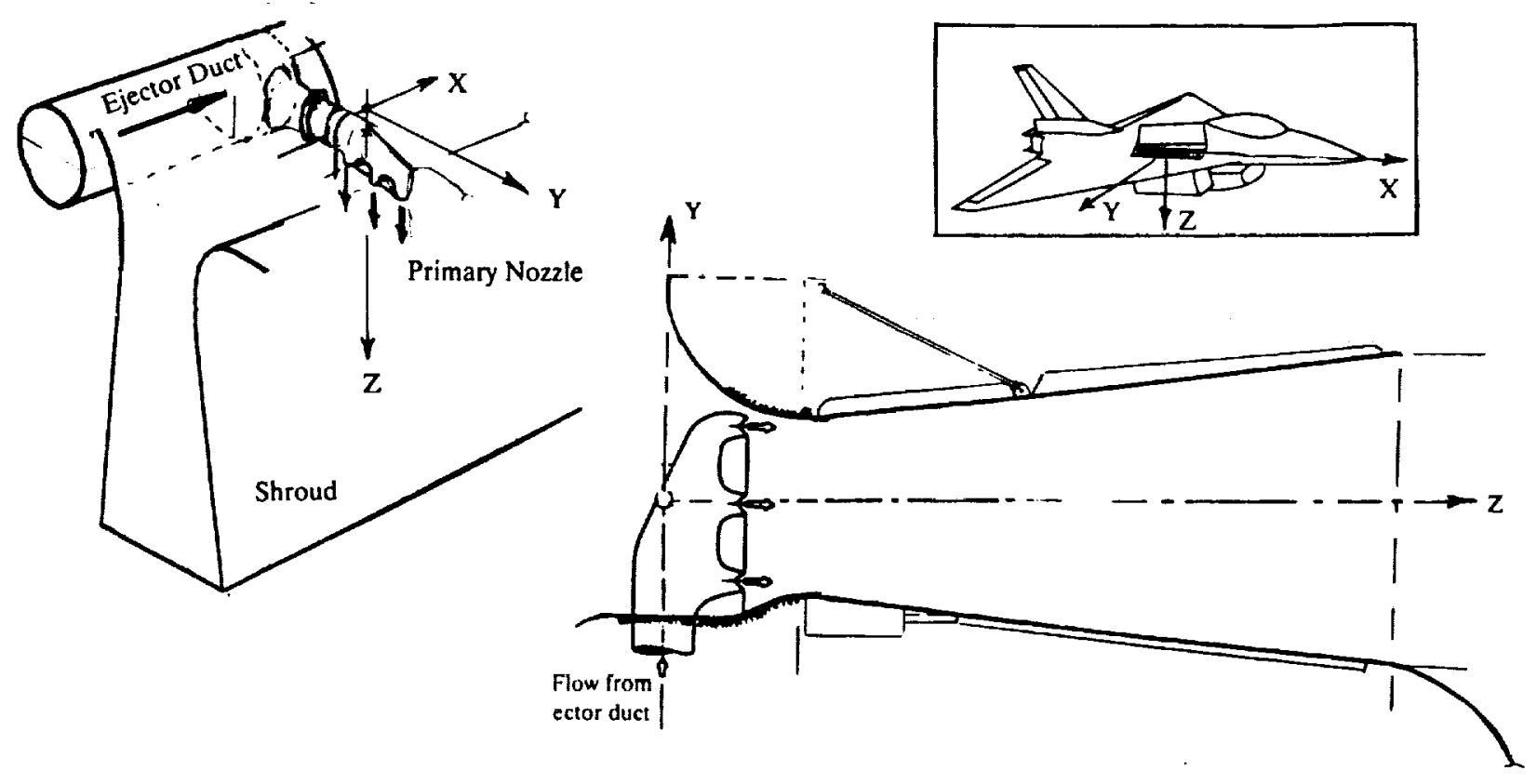

Fig. 1 Ejector configuration for STOVL aircraft 


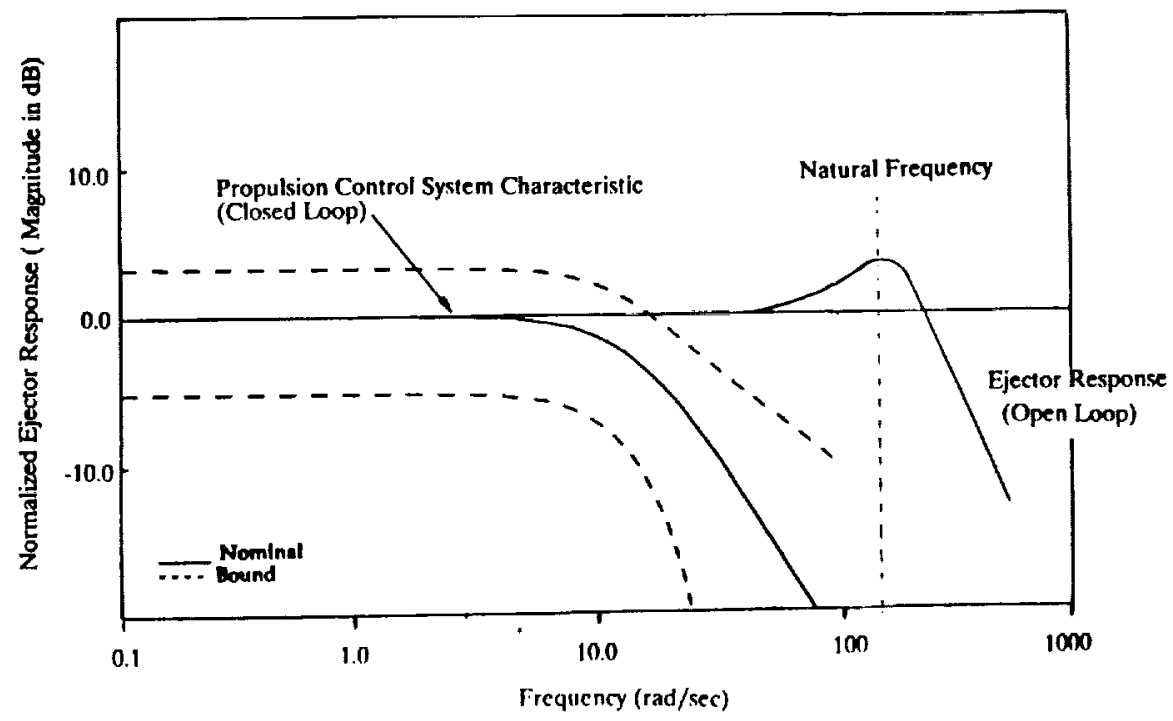

Fig. 2 Response of ejector that can be approximated by quasi-steady model

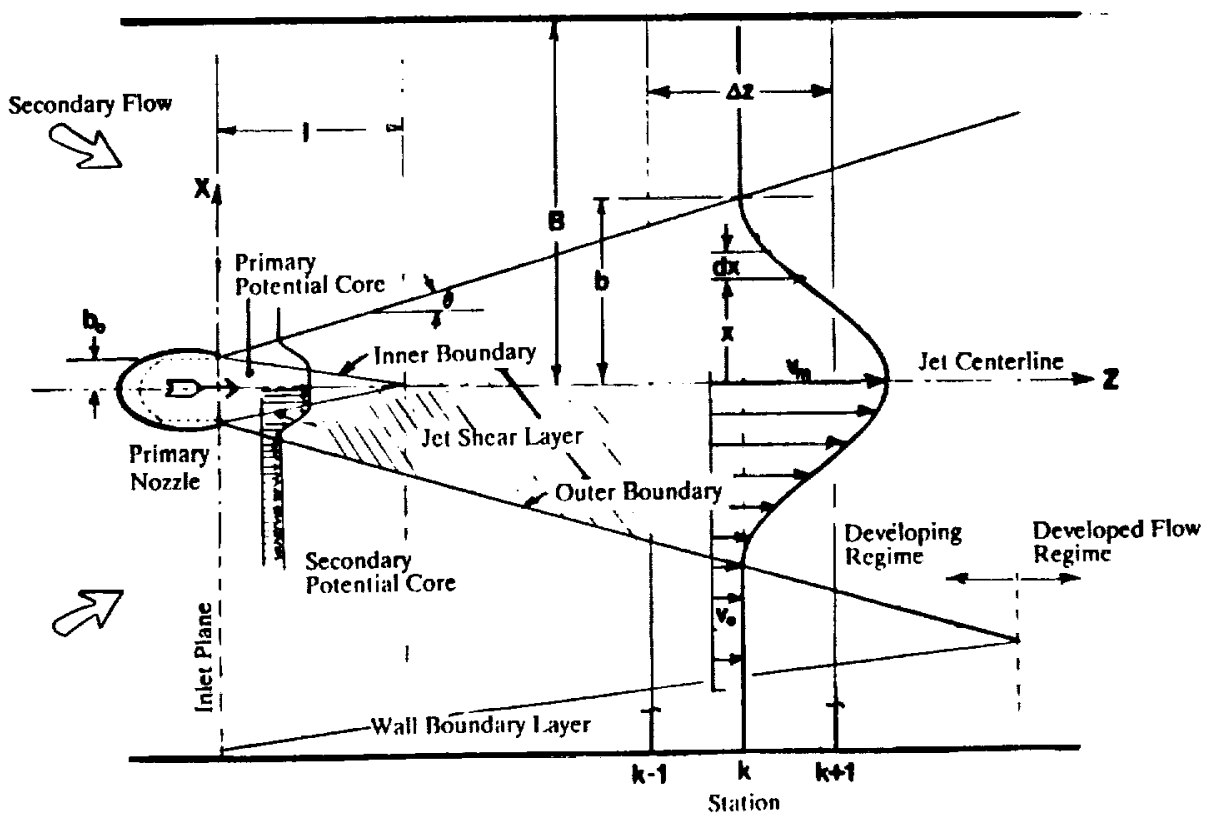

Fig. 3 Features of the mixing region

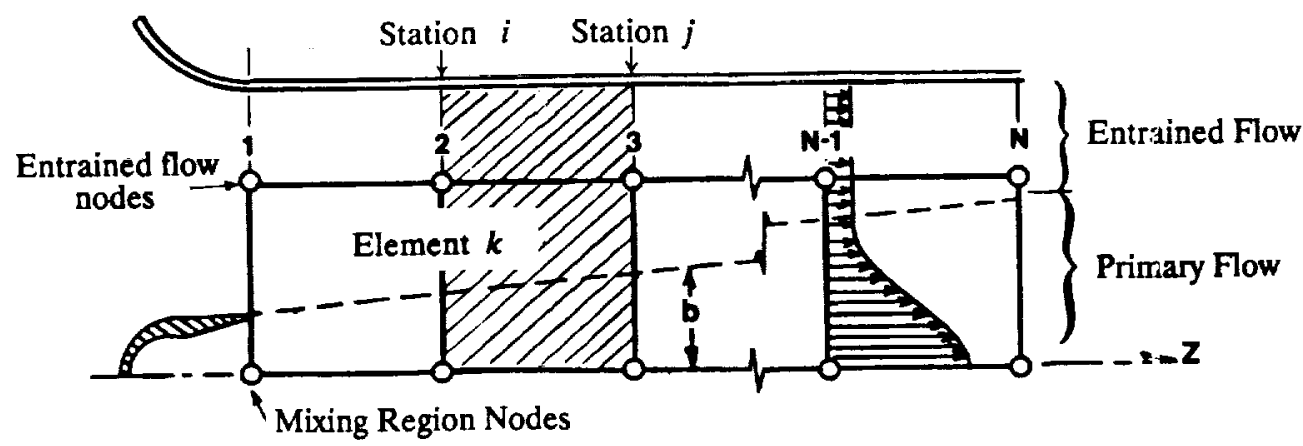

Fig. 4 Computational grid 


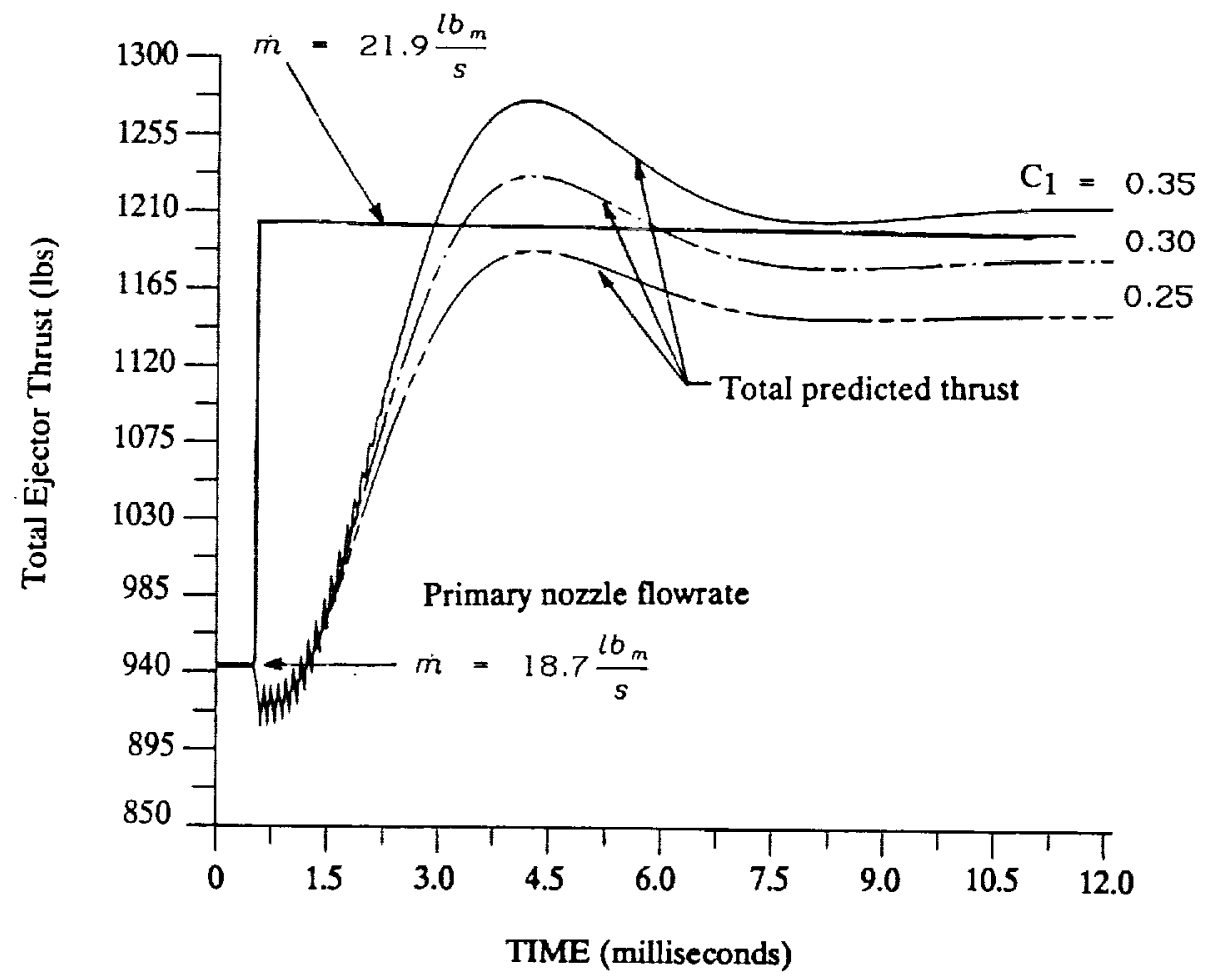

Fig. 5 Result from ejector transient flow test case

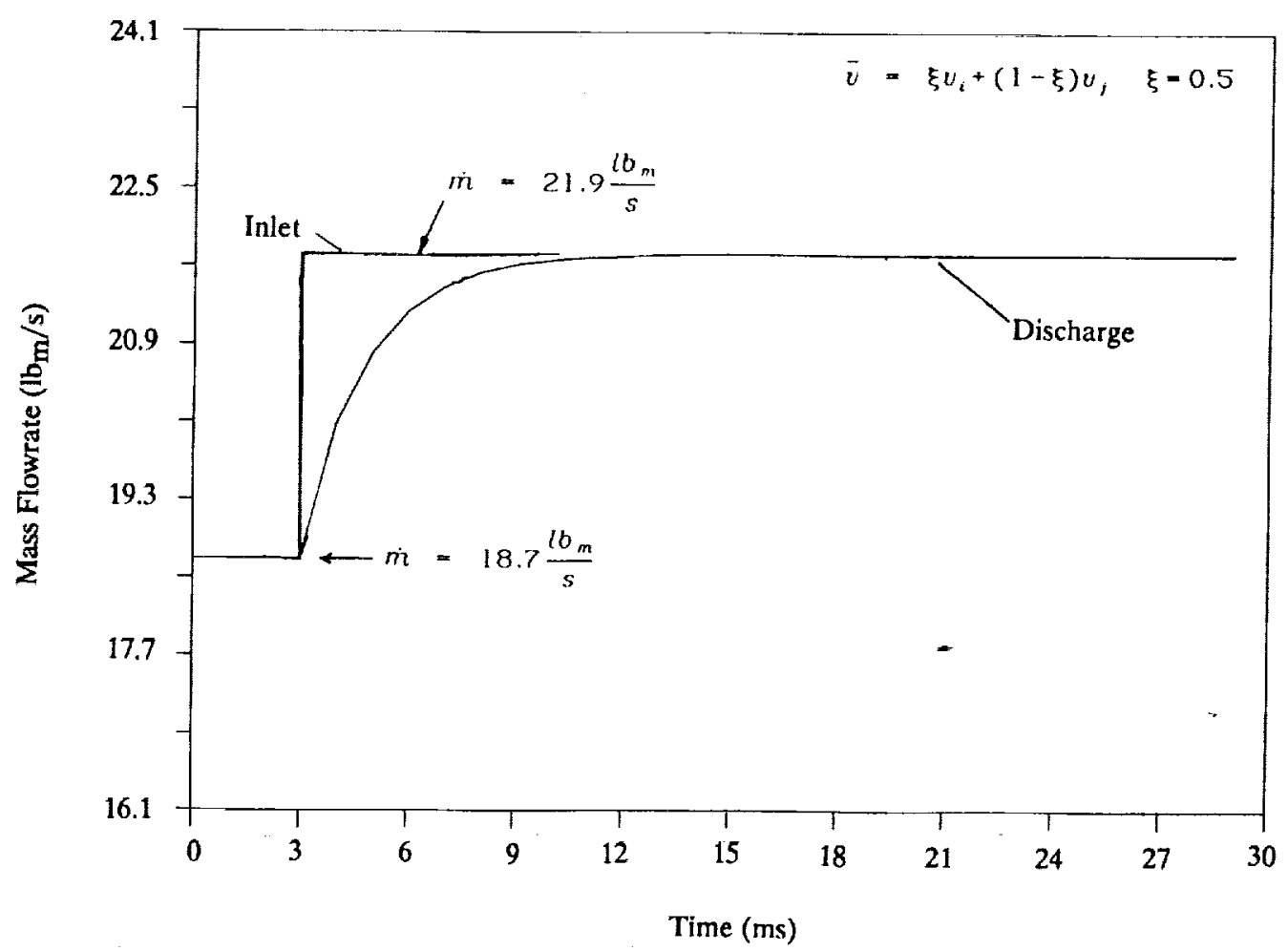

Fig. 6 Result from duct transient flow test case 


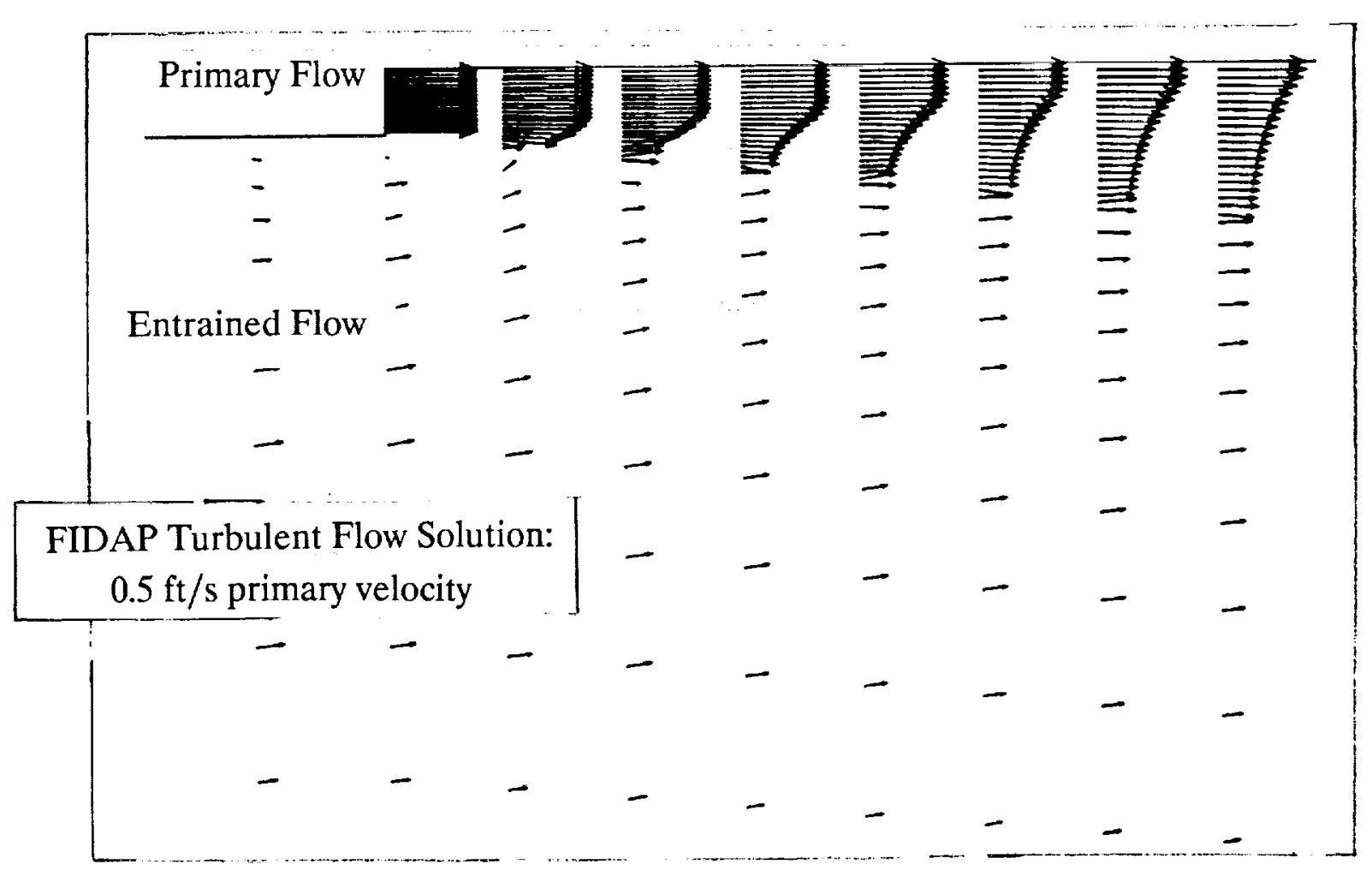

a) Mixing region velocity predictions

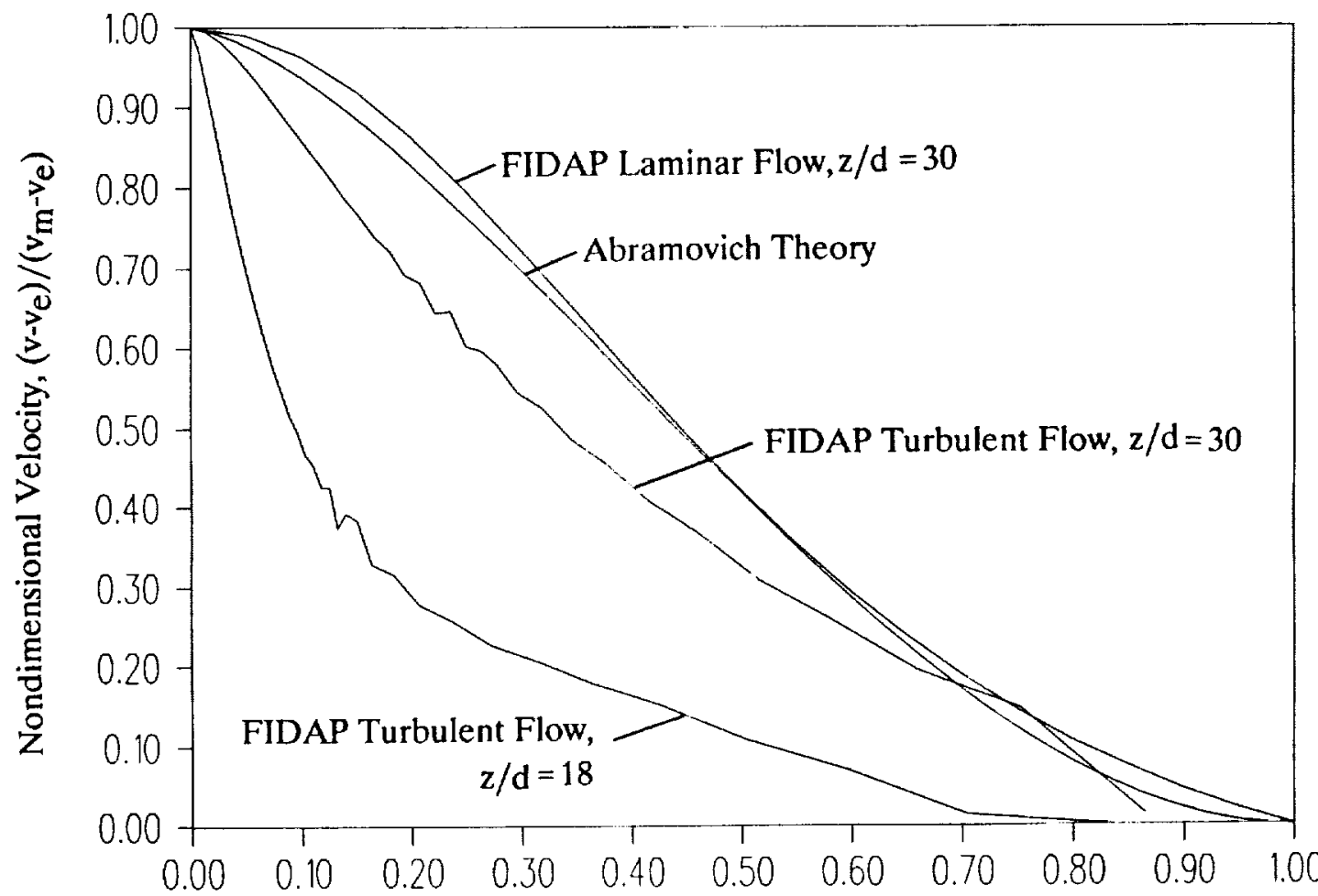

Nondimensional Transverse Position, $\mathrm{x} / \mathrm{b}$

b) Self-similar velocity profiles

Fig. 7 CFD velocity predictions 


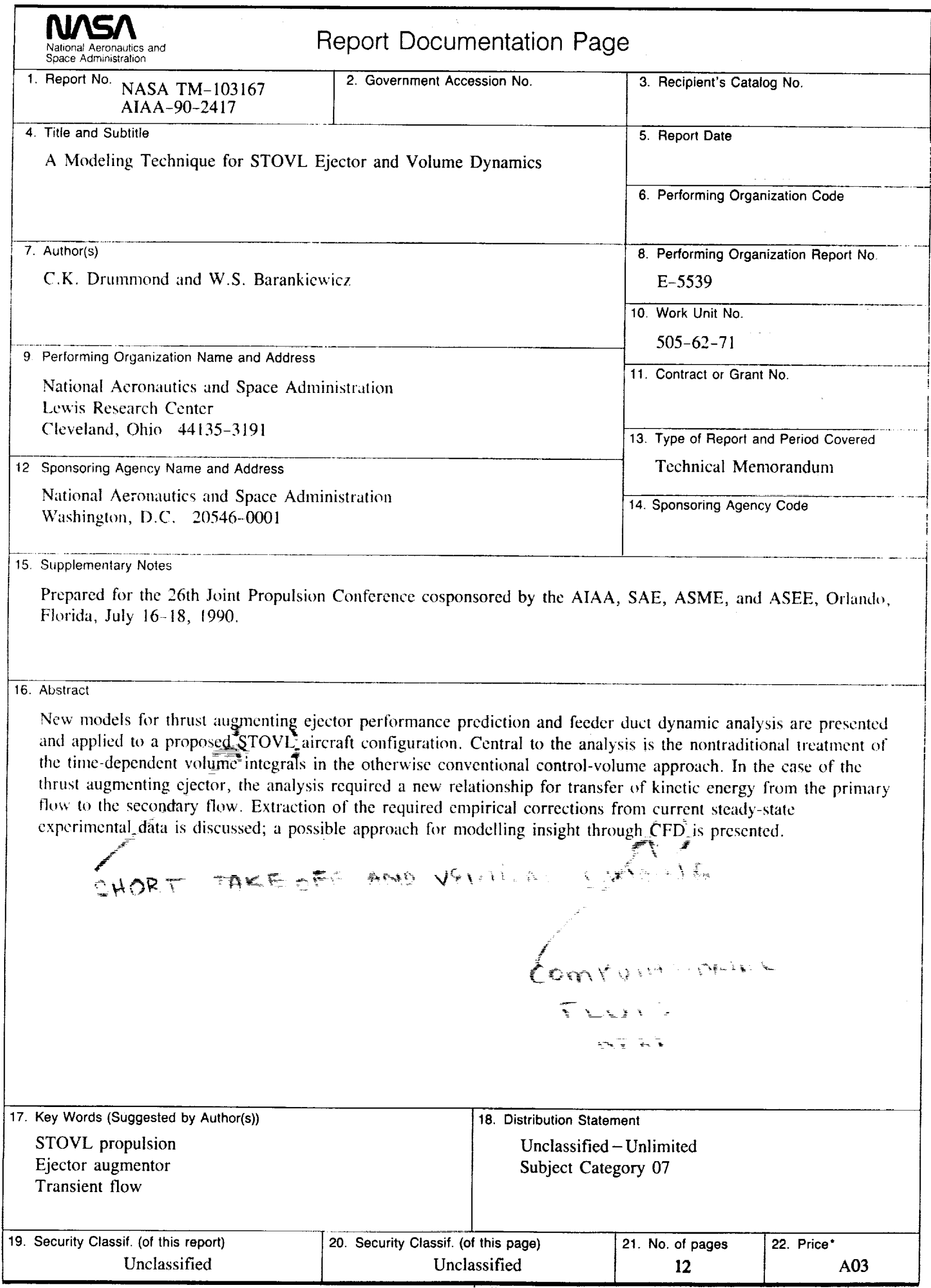

